\title{
Escala Multi-fatorial de Individualismo e Coletivismo: Elaboração e Validação de Construto ${ }^{1}$
}

\author{
Valdiney V. Gouveia², Josemberg M. de Andrade, \\ Girlene R. de Jesus, Maja Meira e Nilton F. Soares \\ Universidade Federal da Paraíba
}

\begin{abstract}
RESUMO - O objetivo deste estudo foi apresentar uma medida multi-fatorial de atitudes individualistas e coletivistas, comprovando sua validade de construto e convergente. Partiu-se da tipologia de Triandis (1995), acrescentando dois novos componentes destes construtos: o protoindividualismo e o individualismo expressivo. Participaram do estudo 304 pessoas, a maioria do sexo feminino (62,5\%), com idade média de 29 anos. Estes responderam a Escala Multi-Fatorial de Individualismo e Coletivismo (EMIC), a Escala de Identificação Endogrupal e uma lista de variáveis demográficas. Comprovou-se a estrutura multi-fatorial da EMIC através de uma análise fatorial confirmatória, que revelou índices aceitáveis de bondade de ajuste $\left(\chi^{2} /\right.$ g.l. $=2,38, A G F I=0,85$ e RMSEA $=0,07$ ). Os Alfas de Cronbach das subescales se situaram entre 0,34 (individualismo horizontal) e 0,68 (coletivismo horizontal). Todos os fatores de individualismo e coletivismo se correlacionaram diretamente com o seu respectivo atributo teórico; a única correlação não significativa foi do individualismo expressivo com o atributo pessoal expressivo $(r=0,08, p>0,05)$. Concluiu-se que, comparando com medidas prévias, esta se mostrou adequada, embora se recomende a elaboração de novos itens para contemplar a dimensão do individualismo horizontal.
\end{abstract}

Palavras-chave: individualismo; coletivismo; atitudes; identificação endogrupal.

\section{Multi-factorial Scale of Individualism and Collectivism: Elaboration and Validity}

\begin{abstract}
This study aimed at introducing a multi-factorial measure of individualist and collectivist attitudes, by checking its construct and convergent validities. We departed from the Triandis' typology (1995), adding two new components of these constructs: protoindividualism and expressive individualism. 304 respondents participated in this study, most of them being woman (62.5\%), with mean age around 29 years. They answered the Multi-Factorial Scale of Individualism and Collectivism (MSIC), the In-Group Identification Scale and a list of demographic variables. The multi-factorial structure of the MSCI was proved through a confirmatory factor analysis, which revealed acceptable goodness of fit indexes $\left(\chi^{2} / \mathrm{g} .1 .=2.38, A G F I=\right.$ .85 , and RMSEA = .07). The Cronbach's Alphas for the factors ranged from .34 (horizontal individualism) to .68 (horizontal collectivism). All factors of individualism and collectivism were correlated directly with its respective theoretical attribute; the only non significant correlation was that of the expressive individualism with the expressive personal attribute $(r=.08, p>$ $.05)$. We then concluded that, compared with previous measures, this one showed itself appropriate, although the elaboration of new items is recommended to contemplate the dimension of horizontal individualism.
\end{abstract}

Key words: Individualism; Collectivism; Attitudes; In-Group Identification.

A tentativa de explicar as relações que se estabelecem entre os homens (individualmente ou em grupo) e a sociedade como um todo integrado tem sido empreendida em diferentes âmbitos das Ciências Humanas e Sociais, produzindo algumas dicotomias clássicas na literatura. Por exemplo, Tönnies (1887 / 1979) propôs a tipologia comunidade vs. associação, baseado na vontade natural e na vontade racional, respectivamente, para descrever a estrutura social de sua época. Durkheim (1893 / 1982), por sua vez, falava em solidariedade mecânica vs. solidariedade orgânica, indicando que a sociedade, no seu estado inicial, seria construída

1 O presente estudo compreende parte de um projeto integrado, financiado pelo CNPq (Proc. CNPq - 520521/99-4). Os autores agradecem a esta instituição.

2 Endereço: Universidade Federal da Paraíba, CCHLA - Depto. de Psicologia, Programa de Pós-Graduação em Psicologia Social - 58059900 João Pessoa, Pb. E-mail: vgouveia@cchla.ufpb.br ou vvgouveia @uol.com.br. em função das semelhanças dos membros no coletivo e, com o passar do tempo, seria como um organismo biológico com seus membros mais autônomos e ao mesmo tempo interdependentes. Os estudos sobre tradição vs. modernidade, neste contexto, parecem ter resumido antigas aspirações, propondo dois padrões de homem e de organização social que explicariam a própria evolução da sociedade (Gusfield, 1967).

A última dicotomia antes citada foi predominantemente estudada durante os anos 60 e 70, quando começaram a ter lugar os "milagres econômicos" em alguns países, como no México e Brasil, fazendo entrever um homem que se modernizava (Gouveia, 1998). Não obstante, por sua natureza temporal e definida em função de dados econômicos dos países, começou a perder força explicativa nos anos subseqüentes. Não demorou para que os pesquisadores se dessem conta de que o padrão moderno era uma falácia, um artefato ou uma invenção que estava predestinada a sucumbir e dar 
lugar a tradição que logo seria suplantada por uma nova ordem moderna (Gusfield, 1967). Hoje em dia, por exemplo, já não se fala no moderno, mas no pós-moderno (Touraine, 1993). Recuperando as idéias desta "teoria da modernidade", porém procurando enfatizar mais aspectos psicológicos e sociais da relação do indivíduo com a sociedade, Hofstede (1984) propôs uma nova dicotomia: individualismo vs. coletivismo.

\section{Os construtos individualismo e coletivismo}

O individualismo e o coletivismo se constituíram nas duas últimas décadas em um verdadeiro programa de investigação (Gouveia \& Clemente, 1998; Lonner \& Berry, 1994). Embora possam ser identificados fatores que os caracterizavam antes dos anos 80 (Braithwaite \& Scott, 1991), foi a partir de então quando se fizeram mais populares. Hofstede (1984) foi o grande responsável por sua difusão, identificando uma estrutura unidimensional que expressaria a "(in) dependência emocional de grupos, organizações e outras coletividades" (p. 157).

No seu estudo original, este autor considerou as respostas de aproximadamente 100.000 empregados da empresa IBM para um questionário que incluía 14 metas do trabalho; estas permitiram derivar duas dimensões básicas: masculinidade-feminilidade e individualismo-coletivismo. Esta última é a que interessa aqui. Através de uma análise de Componentes Principais foram identificados seis itens que permitiam descrevê-la: tempo pessoal $(+)$, liberdade $(+)$, desafio $(+)$, capacidade (-), condições físicas (-) e uso de habilidades (-). Aqueles com saturações fatoriais positivas (+) correspondiam ao individualismo, ao passo que as com saturações negativas (-) contemplariam o coletivismo.

Para Hofstede (1984), em termos conceituais, esta dimensão compreende uma preferência por contextos sociais pouco fechados, onde se supõe que são os indivíduos que têm que cuidar de si mesmos e apenas de sua família nuclear, frente a uma dependência aos grupos dos quais os indivíduos fazem parte. Países com máxima pontuação no Índice de Individualismo seriam tidos como individualistas (por exemplo, Estados Unidos, Canadá), enquanto que aqueles como baixa pontuação receberiam a denominação de coletivistas (por exemplo, Venezuela, Paquistão). Apesar desta ênfase nestes construtos como pólos de uma mesma dimensão, percebeu-se prontamente que, ao menos desde uma análise mais psicológica, como o próprio autor sugere (Hofstede, 1994), uma estrutura multidimensional seria mais apropriada.

Como são atualmente concebidos, o individualismo e o coletivismo podem coexistir em uma mesma pessoa ou cultura (Schwartz, 1990; Triandis, 1995). É possível encontrar pessoas individualistas em culturas coletivistas e vice-versa, ou simplesmente culturas em que se evidencia uma mistura destes dois tipos de orientações, como ocorre na Índia (Sinha \& Tripathi, 1994). Seja como for, reconhece-se que estes construtos expressam síndromes culturais, evidenciando crenças, sentimentos, atitudes, valores etc. que as pessoas de determinada cultura compartilhariam (Triandis, 1993), podendo ser úteis para explicar diferenças culturais no comportamento social (ver Gouveia \& Ros, 2000; Imamoglu, 1998).

Harry C. Triandis representa o grupo de pesquisadores que na Psicologia podem ser mais diretamente relacionados com o tema em questão. Este parece ter recuperado seus estudos dos anos 70 sobre tradição e modernidade, e ajustado às dimensões individualismo e coletivismo. Se antes de Geert Hofstede falava-se em individualismo-coletivismo como uma única dimensão bipolar, a partir desse autor se passou a pensar em individualismos e coletivismos (Triandis, 1995). Triandis não só reconhece que não se trata de uma única dimensão bipolar, senão que estes construtos podem ser representados com ênfase em diferentes atributos das orientações sociais, como a justiça social ou a hierarquia (Gouveia \& Clemente, 1998). Resumindo algumas das definições apresentadas por Triandis e seus colaboradores, seria possível entender estes construtos nos seguintes termos (ver também Gouveia \& Clemente, 2000):

Individualismo. Enfatiza a idéia de que são os indivíduos, enquanto seres autônomos, a unidade principal de sobrevivência. $\mathrm{O}$ indivíduo está por cima dos grupos em todos os aspectos, o que implica geralmente na separação em relação aos familiares e uma ruptura com os ancestrais. Tal padrão de orientação expressa uma tendência a desejar o êxito, a valorizar sua própria intimidade. As relações pessoais, embora mais freqüentes, são geralmente contratuais. $\mathrm{O}$ individualista pensa, sente e atua segundo seus próprios interesses, importando em menor medida o contexto social em que se encontra.

Coletivismo. Contempla a noção de que os grupos são a unidade de sobrevivência, sendo os indivíduos uma parte inseparável destes. Os grupos são mais importantes do que a própria pessoa, podendo ser reduzidos em número, porém grandes em extensão (por exemplo, a família com todos os seus ascendentes e descendentes). As pessoas que assumem este padrão de orientação mantêm fortes relações com os demais membros do seu endogrupo, compartilhando freqüentemente os mesmos interesses. Contempla-se uma tendência à cooperação e ao cumprimento com os demais.

Com estas duas grandes definições em mente e recuperando a clássica dimensão distância de poder - expressa à medida em que os membros de uma sociedade aceitam como legítimo que o poder das instituições e organizações esteja desigualmente distribuído (Hofstede, 1984) - Triandis (1994, 1995) identificou dois atributos principais do individualismo e do coletivismo: horizontal e vertical. Este autor reconhece que podem existir outras facetas destes construtos, porém as apresenta como sendo as mais fundamentais, e propõe diferenciar as pessoas e culturas nesses termos. Por exemplo, considera que o individualismo norte-americano (vertical) é diferente do Sueco (horizontal), ou que o coletivismo coreano (vertical) é diferente daqueles dos kibutz israelitas (horizontal) (Triandis \& Gelfand, 1998). Uma definição de cada uma das quatro dimensões resultantes pode ser encontrada em Triandis (1995), como se resume a seguir: 
O atributo horizontal sublinha que as pessoas são similares na maioria dos aspectos, especialmente no status. No caso do individualismo, em função da alta liberdade e igualdade que estas experimentam, constrói-se um eu independente, mas não diferente dos demais membros da sua cultura; em outras palavras, as pessoas que se orientam pelo individualismo horizontal querem ser distintas dos grupos e são auto-confiantes; estas estão prontas para dizer: "eu quero fazer minhas próprias coisas". Em relação ao coletivismo, contempla-se uma baixa liberdade, porém alta igualdade, explicando o motivo de um eu interdependente e compartilhado com os demais membros da sociedade. Assim, as pessoas se percebem como sendo similares às outras, enfatizam objetivos comuns com os outros, buscam a interdependência e a sociabilidade, embora não sejam submetidas facilmente à autoridade.

O conceito vertical põe ênfase em aceitar a desigualdade e privilegiar a hierarquia. Para os individualistas, isto se reflete em um eu independente e diferente dos demais; aceitase a baixa igualdade ao passo que é dada máxima importância ao sentido de liberdade, definindo uma típica democracia de mercado. As pessoas que seguem esta orientação querem ser distintas adquirindo status social; fazem isto em competições individuais com outros, estando prontas para dizer: "eu quero ser a melhor". No caso dos coletivistas, traduz-se no sentido de servir ao grupo, fazer sacrifícios para o benefício do seu próprio grupo afiliativo e cumprir suas obrigações impostas como normas sociais. Contempla-se um eu interdependente mas diferente dos demais, possivelmente devido ao baixo sentido de liberdade e igualdade.

Levando-se em consideração estes aspectos, Triandis (1995) identifica uma característica principal que melhor descreve a pessoa que adota cada um destes tipos de orientação, a saber: individualismo horizontal $\rightarrow$ ser único; individualismo vertical $\rightarrow$ orientado ao êxito; coletivismo horizontal $\rightarrow$ ser cooperativo; e, coletivismo vertical $\rightarrow$ ser servidor. Os atributos horizontal e vertical de cada construto podem se correlacionar diretamente entre si; estes configurariam uma mesma dimensão de ordem superior, correspondendo ao individualismo ou coletivismo (Gouveia, Clemente \& Espinosa, 2001).

Não há dúvida de que o modelo de Triandis representa na atualidade um avanço na compreensão do individualismo e do coletivismo. Porém, o próprio autor sugere alguns aspectos que exigem novas contribuições. Por exemplo, comenta que seria necessário elaborar novos itens para representar sua dimensão individualismo vertical, atualmente limitada ao conceito de competição; considera que deveria igualmente incluir noções como ser distinto, destacar-se da maioria, ser famoso e ter poder (Triandis \& Gelfand, 1998). Gouveia e Clemente (1998) também sugerem que se revise a dimensão individualismo horizontal; já na escala de Singelis, Triandis, Bhawuk e Gelfand (1995), a correlação elemento-total indica a existência de um sentido específico deste fator, não sendo limitado à idéia de ser único.

Certamente estes aspectos precisam ser contemplados em futuras pesquisas, porém não são os únicos. Além disso, as dimensões propostas não cobrem todos os sentidos do individualismo e do coletivismo (Triandis, 1995). Talvez sejam mais aplicadas na explicação do comportamento em sociedades industriais avançadas, usando-se uma expressão de Ronald Inglehart. Em culturas como a brasileira, com claras desigualdades sócio-econômicas, talvez fosse necessário fazer uso de um construto apresentado por Triandis (1988): o protoindividualismo, que é típico das sociedades onde as pessoas realizam suas atividades muitas vezes com independência das demais, sendo os caçadores e pescadores personagens representativos desta orientação. Este tipo de individualismo parece ser mais uma forma de sobreviver, não de relacionar-se com outras pessoas (Gouveia, 1998). Indivíduos protoindividualistas, ao contrário do que se pensa, não se encontrariam apenas em ambientes rurais; devido a escassez de recursos, o meio urbano também favoreceria o desenvolvimento deste tipo de orientação. Não se descarta também a importância que o construto individualismo expressivo possa ter neste contexto; Parsons (1959 / 1976) o identifica como típico de hispano-américa, enfocando uma tendência a centrar-se em torno dos pontos de referência relacionais, especialmente a comunidade local, desestimando as orientações instrumentais.

Finalmente, o individualismo e coletivismo apresentam ao menos cinco atrativos no âmbito dos estudos intra e interculturais, a saber (Kagitçibasi, 1994): (a) permitem diferenciar sistematicamente as sociedades; (b) produzem um padrão de conduta coerente com a orientação cultural; as pessoas de culturas individualistas tendem a apresentar atitudes, valores e condutas individualistas, enquanto as coletivistas fazem o mesmo em termos coletivistas; (c) esta diferença pode ser encontrada também em outros processos psicológicos, permitindo fazer predições para uma variedade de condutas; (d) além das diferenças culturais, o nível individual e o coletivo apresentam variabilidade intracultural, o que se faz útil para a explicação das diferenças indivíduogrupo em relação a várias características psicológicas; e, (e) seu potencial para se converter em dimensões universais de variação das culturas.

Tomando como referência estes atrativos mencionados, muitos esforços têm sido empreendidos para se desenvolver teorias a respeito destes construtos (Gouveia, 1998; Gouveia \& Clemente, 1998), havendo no entanto muitas especulações e conhecimentos enviesados (Kagitçibasi, 1997). Triandis (1995), por exemplo, caracteriza a sociedade brasileira como orientada ao coletivismo vertical, sem ter dados concretos como base. Definitivamente, as especulações sobre os tipos de orientações devem ser contrastadas com dados de pesquisas empíricas. Neste contexto, faz-se necessário atentar para as escalas de medida disponíveis na literatura, analisando as metodologias empregadas com o fim de tentar suprir possíveis carências que tornem a validade destas duvidosa, reforçando antigas especulações.

\section{Medidas prévias do individualismo e do coletivismo}

- Índice de Individualismo. Proposto por Hofstede (1984), 
este índice reúne itens que avaliam o coletivismo (capacitação, condições físicas e uso de habilidades) e o individualismo (tempo pessoal, liberdade e desafio), os quais são organizados em um único fator bipolar. Foi elaborado para avaliar o quanto as culturas poderiam se diferenciar em termos desta orientação, sendo a maior pontuação indicação de individualismo e a menor de coletivismo. Não se encontraram informações sobre sua consistência interna; comprovou-se sua validade convergente ao se demonstrar que estava diretamente correlacionado com os valores vida excitante e um mundo de beleza (Hofstede \& Bond, 1984). Gouveia e Ros (2000) mostraram que este também estava correlacionado com os valores culturais autonomia $(r=0,68, p<0,01)$ e conservação $(r=-0,61, p<0,01)$.

- Questionário INDividualismo-COLetivismo. Compreendeu um esforço por desenvolver uma medida multifatorial do individualismo e coletivismo dos indivíduos (Hui, 1988). Centra-se na relação de (in)dependência do indivíduo com o grupo, avaliando oito potenciais grupos de pertença: família, conhecidos, cônjuge, pais, parentes, vizinhos, amigos e companheiros de trabalho. A versão original se compõe de 63 itens, existindo uma adaptada que está formada por 36 itens (Hui \& Yee, 1994). Os seus autores apresentam algumas provas sobre sua adequação psicométrica; os Alfas de Cronbach das oito subescalas estão entre 0,46 (cônjuge) e 0,76 (parentes). Tem sido sugerido trabalhar com sua pontuação total, denominada Índice de Coletivismo Geral, cuja informação sobre sua consistência interna não foi disponibilizada. Sua validade convergente foi comprovada com as pontuações de interesse social ( $r$ médio $=0,33, p<0,01) \mathrm{e}$ necessidade de aprovação $(r=0,25, p<0,05)$, bem como com os motivos de afiliação $(r=0,44, p<0,001)$ e autonomia $(r=-0,36, p<0,001)$ (Hui, 1988).

- Escala de Individualismo-Coletivismo. Esta mede o individualismo-coletivismo, focalizando os interesses pessoais versus grupais num contexto de grupos de trabalho; são estabelecidas diferenças entre crenças, valores e normas para descrever a citada dimensão (Wagner \& Moch, 1986). Inicialmente, foram selecionados 34 itens para compor a referida escala, realizou-se uma análise fatorial exploratória com rotação varimax, tendo permanecido onze itens em função das cargas fatoriais. Seus autores também efetuaram uma análise fatorial confirmatória, obtendo lambdas (cargas fatoriais) estatisticamente diferentes de zero $(t>1,96)$; os Alfas de Cronbach dos seus atributos principais foram os seguintes: crenças, 0,84; valores, 0,67; e normas, 0,80. Moorman e Blakely (1995) também realizaram uma análise fatorial confirmatória desta escala, tendo observado índices de bondade de ajuste satisfatórios: $C F I=0,92$ e $T L I=0,90$.

- Questionário de Individualismo-Coletivismo. Elaborado por Triandis, com a colaboração de 15 pesquisadores de nove países, este foi inicialmente composto por 21 itens característicos de culturas individualistas e coletivistas. Realizada uma análise de Componentes Principais, com rotação varimax, tal questionário ficou reduzido a 17 itens, representados em quatro fatores com saturações iguais ou superiores a 0,30 , a saber: I - autoconfiança com hedonismo, II - separação do endogrupo, III - integridade familiar e IV - interdependência e sociabilidade (Triandis \& cols., 1986). Sua validade convergente foi comprovada com o Índice de Individualismo (Hofstede, 1984), verificando-se correlações dos seus fatores na ordem 0,67 a $0,73(p<0,05)$. Os Alfas para os seus fatores estiveram entre 0,58 e 0,61 (Feldman \& Rosenthal, 1991).

- Questionário dos Três Fatores. Este é formado por 29 itens, distribuídos nos seguintes fatores: autoconfiança com competição, consideração para o endogrupo e distância de endogrupos. Seus autores não apresentam informações sobre sua consistência interna; Morales, López e Vega (1992) observaram na Espanha que o conjunto total dos itens obteve um Alfa de Cronbach de 0,74. Através de uma análise fatorial, estes autores chegaram a uma estrutura com quatro dimensões cujos itens apresentaram carga fatorial igual ou superior a 0,30 , explicando $35,5 \%$ da variância total; tais fatores foram denominados de auto-suficiência com busca de êxito, interdependência e ajuda mútua, auto-atribuição dos próprios êxitos e independência emocional em relação ao endogrupo e independência na eleição de parceiros e não aceitação de responsabilidade nos fracassos das pessoas do endogrupo. Pouco se sabe sobre a consistência interna destes fatores.

- Escala de Coletivismo. Esta é composta por dez itens que expressam condutas grupais. Yamaguchi (1994) apresenta os coeficientes de consistência interna variando de 0,77 a 0,88 , dependendo da amostra. Um teste-reteste no intervalo de três meses $(\mathrm{N}=75)$ apoia a precisão desta escala $(r=0,71, p<0,01)$. Claramente são percebidas suas vantagens: é uma medida curta (reúne poucos itens) e de fácil aplicação. Não obstante, talvez não seja tão eficaz no âmbito das relações pessoa-pessoa, com independência do grupo de pertença. Esta é definida como uma medida unidimensional, sendo as baixas pontuações interpretadas como individualismo e as altas como coletivismo, tal como ocorre com o instrumento de Hofstede (1984). Porém, é preciso lembrar que sua proposta é medir estes construtos no nível individual de análise; o próprio Hofstede (1994) sugere que neste nível uma estrutura multidimensional seria mais adequada.

- Escala de Auto-Imagem. Segundo Singelis (1994), os itens desta escala foram elaborados para medir a constelação de pensamentos, sentimentos e ações que contemplam as duas construções do eu: independente e interdependente. Para definir a versão final da escala, considerou-se um conjunto de 45 itens, os quais foram submetidos a uma análise de Componentes Principais; foram eliminados os itens com saturações inferiores a 0,35 , restando 12 em cada um dos dois fatores que teoricamente avalia. Uma análise fatorial confirmatória indicou a adequação deste modelo $\left(\chi^{2} /\right.$ g.l. $\left.=1,87 ; A G F I=0,77\right)$. Os 
seus coeficientes de consistência interna (Alfa de Cronbach) foram 0,74 e 0,70 , para os fatores interdependente e independente, respectivamente. Uma limitação evidente desta medida é a ênfase em dois únicos componentes, que inclusive podem corresponder ao individualismo (independência) e coletivismo (interdependência), ressaltando principalmente seu atributo horizontal (Gouveia \& Clemente, 1998).

- Escala de Individualismo e Coletivismo Horizontal-Vertical. Sua elaboração tomou como referência um conjunto de 94 itens, alguns reunidos de pesquisas prévias de Triandis e cols. (1995) e outros elaborados com o fim de cobrir suas quatro dimensões teóricas (ver Singelis, \& cols., 1995). Destes, 70 puderam ser agrupados em tais dimensões. Depois, através de uma análise de Componentes Principais, considerando por separado os itens em função das suas dimensões respectivas, foram eliminados aqueles com baixa carga fatorial (menor do que $\pm 0,30$ ). Finalmente, procurou-se selecionar os 32 itens cujas correlações com o total da escala correspondente foram mais fortes; os índices de consistência interna (Alfa de Cronbach) resultantes foram os seguintes: individualismo horizontal, 0,67; individualismo vertical, 0,74 ; coletivismo horizontal, 0,74 ; coletivismo vertical, 0,68 . Esta estrutura multidimensional foi comprovada através de análises fatoriais confirmatórias (Gouveia \& cols., 2001). Singelis e cols. (1995) reuniram provas que suportam a validade de construto desta medida; por exemplo, as pontuações de auto-imagem independente se correlacionaram positivamente com o individualismo horizontal $(r=0,45, p<0,01)$ e negativamente com o coletivismo vertical $(r=-0,26, p<0,01)$. No caso das pontuações em auto-imagem interdependente, estas se correlacionaram diretamente com o coletivismo horizontal $(r=$ $0,43, p<0,01)$ e vertical $(r=0,50, p<0,01)$, sendo inversa sua correlação com o individualismo horizontal $(r$ $=-0,25, p<0,01)$. Apesar das evidências favoráveis a esta escala, Gouveia e Clemente (1998) ressaltam que os itens do individualismo horizontal são muito abrangentes, cobrindo mais do que o atributo "um ser único".

Em resumo, embora possam ser identificados diversos instrumentos para medir o individualismo e o coletivismo, a maioria tem sido elaborada sem um marco teórico sólido de referência; os procedimentos estatísticos exploratórios são indicação deste fato. Neste contexto, a medida apresentada por Triandis e seus colaboradores (Singelis \& cols., 1995; Triandis, 1995; Triandis \& Gelfand, 1998) é bastante proeminente, porém não incorpora dimensões que parecem fundamentais para explicar as condutas de culturas latinas e/ou onde a escassez econômica é evidente, como pode ser o caso do Brasil. Estes aspectos têm guiado o interesse do presente estudo que, partindo da tipologia destes autores, procura incorporar duas dimensões até então não medidas na literatura: protoindividualismo e individualismo expressivo. Basicamente o objetivo é apresentar uma nova medida do individualismo e coletivismo, procurando comprovar sua validade de construto e convergente. Este processo é descrito a seguir.

\section{Escala multi-fatorial de individualismo e coletivismo}

O presente estudo foi realizado em três etapas, a saber: redação e seleção dos itens; sua análise empírica, isto é, comprovação do seu poder discriminativo e validação semântica; e estabelecimento dos parâmetros psicométricos desta escala. As duas primeiras compreendem passos preliminares, sendo portanto aqui descritas sumariamente; a última etapa é considerada a principal, sendo detalhados os aspectos inerentes à comprovação da qualidade métrica da medida em questão.

\section{Elaboração e seleção dos itens}

Tendo identificado previamente as dimensões que deveriam ser operacionalizadas, foram reunidos itens da literatura (Gouveia \& Clemente, 1998; Triandis, 1995; Triandis \& Gelfand, 1998) e construídos outros que atendessem às definições apresentadas. Nesta oportunidade, cinco colaboradores foram solicitados individualmente a redigir dois itens para cada dimensão do individualismo e coletivismo. Estes procedimentos permitiram reunir um total de 60 itens, que posteriormente foram analisados por um grupo de oito colaboradores em termos do seu conteúdo e da adequação e/ou correspondência em relação às dimensões a serem avaliadas. Feito isso, chegou-se a uma versão prévia da medida em questão, composta por 47 itens, que se distribuíram aproximadamente de modo eqüitativo nas seis dimensões teóricas do individualismo e coletivismo

Uma amostra de 20 pessoas, igualmente distribuídas quanto ao sexo, com uma idade mínima de 15 anos, tendo cursado o primeiro grau, participou da análise da validade semântica da versão preliminar da escala com 47 itens. Tais pessoas deveriam expressar sua compreensão de cada item, bem como a possibilidade de respondê-lo segundo a escala de resposta proposta, que compreendia sete pontos, com os seguintes extremos: 1 = Discordo Totalmente e $7=$ Concor do Totalmente. Com base nas observações, exceto três palavras que tiveram que ser substituídas por sinônimos, percebeu-se a adequação do conjunto total dos itens, assim como da escala de resposta proposta.

O passo seguinte foi comprovar em que medida os 47 itens da escala apresentavam poder discriminativo aceitável $(p<0,05)$. Neste sentido, esta versão da escala foi aplicada a 200 pessoas, tanto da população geral como estudantes universitários, com uma média de 26 anos de idade, a maioria solteira $(70,9 \%)$, com curso superior $(67,5 \%)$ e do sexo feminino $(56,7 \%)$. Em função das respostas dos participantes foram formados grupos-critério internos a partir da mediana da soma total dos itens, sendo posteriormente comparadas as médias dos grupos inferior e superior para cada item. Estas análises indicaram que 13 dos 47 itens não atendiam o critério de poder discriminativo fixado. Com o fim de selecionar um número equivalente de itens para representar cada uma das seis dimensões aqui tratadas, foram aproveitados cinco daqueles 13 itens não discriminativos, uma vez que 
estes têm sido identificados na literatura como característicos das suas dimensões correspondentes. Tal procedimento permitiu chegar a uma versão com os 30 melhores itens, cinco por cada dimensão. O leitor interessado, uma vez solicitado aos autores deste estudo, poderá ter acesso ao conjunto total dos itens, às análises estatísticas efetuadas e tabelas correspondentes.

Em resumo, restando comprovar seus parâmetros psicométricos, foi possível chegar a uma nova versão da Escala Multi-Fatorial de Individualismo e Coletivismo, cobrindo de modo eqüitativo suas seis dimensões teóricas.

\section{Parâmetros psicométricos da escala}

Como antes indicado, os objetivos nesta etapa foram comprovar a estrutura da Escala Multi-Fatorial de Individualismo e Coletivismo (validade de construto) e a correlação das suas dimensões com atributos pessoais (validade convergente), definidos na literatura como típicos das pessoas que assumem as orientações correspondentes (Gouveia, 1998; Parsons, 1959 / 1976; Triandis, 1988, 1995). De modo complementar, decidiu-se conhecer em que medida tais orientações se correlacionariam com a identificação com distintos endogrupos (grupos de pertença) ou membros destes (por exemplo, a família, os familiares em geral, os vizinhos). Estes resultados serão mais adiante apresentados, sendo a seguir descrito o método empregado.

\section{Método}

\section{Sujeitos}

Participaram do presente estudo 304 pessoas, tanto provenientes da população geral como estudantes do ensino médio e universitário. A maioria era do sexo feminino $(62,5 \%)$, solteira $(67,4 \%)$, católica $(71,5 \%)$, tendo cursado o ensino médio $(52,2 \%)$, com renda familiar entre $\mathrm{R} \$$ $1.000,00$ e $2.500,00(33,4 \%)$. A idade média dos participantes foi de aproximadamente 29 anos, tendo o mais jovem 13 e o mais velho 88 anos.

\section{Instrumentos}

Os participante responderam um questionário constando de quatro partes:

Escala Multi-Fatorial de Individualismo e Coletivismo. Compôs-se de 30 itens, compreendendo atitudes e/ou opiniões, eqüitativamente distribuídos entre as seis dimensões de que trata esta medida (com exemplos de itens entre parênteses): protoindividualismo (É essencial para mim satisfazer minhas necessidades básicas; Tenho medo de não ter amanhã o que possuo hoje); individualismo expressivo (Para realizar-me, minha profissão tem que ser conhecida e admirada; Prefiro aproveitar o lado divertido da vida do que ficar reivindicando direitos); individualismo horizontal (Minhas decisões não são influenciadas pelos demais; Gosto de ter um espaço íntimo, só meu); individualismo vertical (Vencer é tudo; Os meus planos para o futuro estão acima de qualquer coisa); coletivismo horizontal (Quando agridem algum membro da minha família, sinto-me agredido; Minha felicidade depende daqueles que me rodeiam) e coletivismo vertical (Deveria ser ensinado às crianças colocar o dever antes do prazer; No trabalho ou estudo sempre obedeço as ordens dos meus superiores). Cada um dos itens é respondido em escala de sete pontos, com os seguintes extremos: $1=$ Discordo Totalmente e $7=$ Concordo Totalmente.

Escala de Identificação Endogrupal. Composta por seis endogrupos: família, familiares, companheiros de estudo, vizinhos, namorado (a) ou esposo (a) e amigos. Nesta pedese ao sujeito que indique em que medida ele se identifica com cada um dos grupos citados, respondendo numa escala de cinco pontos, com os seguintes extremos: $0=$ Nada Identificado e 8 = Totalmente Identificado. Esta medida foi previamente utilizada por Coelho Júnior (2001), quem descreve seus parâmetros psicométricos.

Questionário dos Valores Básicos. Compreende um conjunto de 24 itens-valores para os quais o respondente precisa indicar seu grau de importância como um princípio-guia na sua vida, utilizando uma escala de resposta que vai de $0=$ Nada Importante a 8 = Muito Importante (ver Gouveia, 1998; Maia, 2000). Os dados referentes ao mesmo não serão considerados no presente estudo.

Lista de Dados Sócio-Demográficos. Os participantes responderam um conjunto de perguntas sobre características pessoais (sexo, idade etc.) e atributos sócio-econômicos (grau de religiosidade, renda familiar etc.).

\section{Procedimento}

Os aplicadores deram as mesmas instruções, seguindo assim um procedimento padrão. Isso visava garantir um mínimo de respostas enviesadas. As instruções enfatizavam como responder a escala, pedindo-se que as respostas fossem dadas individualmente e que não se deixasse nenhum item em branco. A aplicação dos instrumentos foi realizada em salas de aula da universidade e dos colégios e nas residências das pessoas, no caso destas pertencerem à população geral. Com exceção da lista de características sóciodemográficas, os demais instrumentos foram contrabalanceados, procurando evitar o efeito de exposição da informação. O tempo médio para responder os instrumentos foi de 20 minutos.

\section{Análise dos dados}

O tratamento estatístico descritivo dos dados e o cálculo da consistência interna (Alfa de Cronbach) foram realizados com o programa SPSSWIN. A análise fatorial confirmatória foi realizada com a versão 8 do programa LISREL (Jöreskog \& Sörbom, 1989). Decidiu-se realizar este tipo de análise porque ela permite testar hipóteses específicas sobre a estrutura latente do modelo (por exemplo, número de fatores, cargas fatoriais), apresentando os respectivos indicadores de bondade de ajuste: (1) o goodness-of-fit index (GFI) e 
o adjusted goodness-of-fit index ( $A G F I$ ), que leva em conta os graus de liberdade ( $g . l$. $)$ do modelo relativo ao número de variáveis. Estes expressam o montante relativo das (co)variâncias na matriz de covariância observada que pode ser predita pelo modelo. Segundo Rhee, Uleman e Lee (1996), um $A G F I$ de 0,80 ou mais indica que o modelo é adequadamente ajustado aos dados; (2) a razão $\mathrm{c}^{2} /$ g.l. é um índice subjetivo de bondade de ajuste; um valor inferior a 5,0 indica ajustamento adequado (Byrne, 1989); e (3) o Root Mean Square Error of Approximation (RMSEA), que corresponde à discrepância do modelo em função dos graus de liberdade (indica a fraqueza do ajuste dado um número particular de parâmetros no modelo). Tem sido recomendado o valor 0,05 para indicar um ajuste adequado (Van de Vijver \& Leung, 1997). Trabalhou-se com a matriz de correlações como entrada, sendo utilizado o estimador $U L S$ (Unweighted Least Squares).

\section{Resultados}

Para verificar a hipótese de que a escala de individualismo e coletivismo era multi-fatorial, composta pelos seis fatores previamente indicados, fixou-se a extração da estrutura correspondente, designando os itens que teoricamente pertenciam a cada fator. Os resultados desta análise podem ser vistos na Tabela 1.

Como apresentado nesta tabela, todas as cargas fatoriais foram estatisticamente diferentes de zero $(t>1,96, p<0,05)$, indicando sua adequação no fator teórico correspondente. A menor carga $(0,13)$ correspondeu ao item 27 (É bobagem gastar com comidas e remédios especiais para animais) do Fator III (protoindividualismo), sendo a maior $(0,74)$ atribuída ao item 21 (É fundamental que meu/minha parceiro/a se relacione bem com minha família) do Fator VI (coletivismo horizontal). Este modelo apresentou os seguintes índices de ajuste: $\mathrm{c}^{2} / \mathrm{g} .1 .=2,38, G F I=0,88, A G F I=0,85$ e $R M S E A=$ 0,07. Os índices de consistência interna (Alfa de Cronbach) observados para os seis fatores foram os que se descrevem: Individualismo vertical, 0,66; Individualismo horizontal, 0,34; Protoindividualismo, 0,36; Individualismo expressivo, 0,58; Coletivismo vertical, 0,63; e Coletivismo horizontal, 0,68 .

Com o fim de comprovar a validade convergente da escala supracitada, as pontuações dos participantes para cada um dos seus fatores foram correlacionadas com os atributos pessoais que teoricamente os descreveriam. Os resultados a respeito são apresentados na Tabela 2 .

Considerando os coeficientes de correlação, pode-se observar que, como esperado, todos os atributos pessoais se correlacionaram diretamente com sua dimensão teórica correspondente. Não obstante, um dos coeficientes de correlação não foi estatisticamente significativo, correspondendo ao atributo expressivo que presumivelmente representaria o fator individualismo expressivo $(r=0,08, p>0,05)$. Este se correlacionou mais fortemente com os atributos pessoais $u m$ ser único $(r=0,26, p<0,001)$ e orientado ao êxito $(r=0,24$, $p<0,001)$. As demais correlações hipotetizadas podem ser
Tabela 1. Pesos Fatoriais (1) dos itens nos fatores da Escala Multi-Fatorial de Individualismo e Coletivismo

\begin{tabular}{|c|c|c|c|c|c|c|}
\hline \multicolumn{7}{|c|}{ Fatores } \\
\hline Itens & $\begin{array}{c}\text { IV } \\
(\alpha=0,66)\end{array}$ & $\begin{array}{c}\text { IH } \\
(\alpha=0,34)\end{array}$ & $\begin{array}{c}\text { PI } \\
(\alpha=0,36)\end{array}$ & $\begin{array}{c}\text { IE } \\
(\alpha=0,58)\end{array}$ & $\begin{array}{c}\text { CV } \\
(\alpha=0,63)\end{array}$ & $\begin{array}{c}\mathrm{CH} \\
(\alpha=0,68)\end{array}$ \\
\hline 26. & 0,67 & - & - & - & - & - \\
\hline 17. & 0,65 & - & - & - & - & - \\
\hline 02. & 0,59 & - & - & - & - & - \\
\hline 16. & 0,57 & - & - & - & - & - \\
\hline 29. & 0,41 & - & - & - & - & - \\
\hline 07. & - & 0,32 & - & - & - & - \\
\hline 04. & - & 0,31 & - & - & - & - \\
\hline 25. & - & 0,30 & - & - & - & - \\
\hline 24. & - & 0,26 & - & - & - & - \\
\hline 10. & - & 0,14 & - & - & - & - \\
\hline 14. & - & - & 0,47 & - & - & - \\
\hline 12. & - & - & 0,36 & - & - & - \\
\hline 03. & - & - & 0,33 & - & - & - \\
\hline 20. & - & - & 0,24 & - & - & - \\
\hline 27. & - & - & 0,13 & - & - & - \\
\hline 15. & - & - & - & 0,64 & - & - \\
\hline 09 . & - & - & - & 0,49 & - & - \\
\hline 23. & - & - & - & 0,44 & - & - \\
\hline 05. & - & - & - & 0,41 & - & - \\
\hline 18. & - & - & - & 0,21 & - & - \\
\hline 28. & - & - & - & - & 0,60 & - \\
\hline 06. & - & - & - & - & 0,57 & - \\
\hline 11. & - & - & - & - & 0,55 & - \\
\hline 13. & - & - & - & - & 0,45 & - \\
\hline 30. & - & - & - & - & 0,17 & - \\
\hline 21. & - & - & - & - & - & 0,74 \\
\hline 22. & - & - & - & - & - & 0,56 \\
\hline 08. & - & - & - & 一 & - & 0,54 \\
\hline 01. & - & - & - & - & - & 0,51 \\
\hline 19. & - & - & - & - & - & 0,45 \\
\hline
\end{tabular}

Nota: Identificação dos fatores: IV = Individualismo Vertical, $\mathrm{IH}=$ Individualismo Horizontal, $\mathrm{PI}=$ Protoindividualismo, $\mathrm{IE}=$ Individualismo Expressivo, $\mathrm{CV}=$ Coletivismo Vertical, e $\mathrm{CH}=$ Coletivismo Horizontal.

Tabela 2. Validade Convergente do Individualismo-Coletivismo com os Atributos Pessoais

\begin{tabular}{lccllll}
\hline \multicolumn{7}{c}{ Atributos } \\
\hline & Cooperador & Único & Exito & Cumpridor & Expressivo & Batalhador \\
\hline $\mathrm{CH}$ & $0,24^{* * * *}$ & $-0,09$ & 0,11 & $0,35^{* * * *}$ & $0,39^{* * *}$ & $0,25^{* * *}$ \\
\hline $\mathrm{IH}$ & 0,01 & $0,30^{* * *}$ & $0,13^{*}$ & $-0,02$ & 0,05 & 0,05 \\
\hline $\mathrm{IV}$ & $0,19^{* * *}$ & $0,24^{* * *}$ & $0,30^{* * *}$ & $0,18^{* *}$ & $0,15^{*}$ & $0,22^{* * *}$ \\
\hline $\mathrm{CV}$ & $0,22^{* * *}$ & $-0,02$ & $0,20^{* * *}$ & $0,37^{* * *}$ & $0,33^{* * *}$ & $0,26^{* * *}$ \\
\hline $\mathrm{IE}$ & 0,03 & $0,26^{* * *}$ & $0,24^{* * *}$ & 0,06 & 0,08 & 0,06 \\
\hline $\mathrm{PI}$ & $0,18^{* *}$ & $0,12^{*}$ & $0,19^{* * *}$ & $0,18^{* *}$ & $0,19^{* * *}$ & $0,19^{* *}$ \\
\hline
\end{tabular}

Notas: $* p<0,05 ; * * p<0,01 ; * * * p<0,001$. Identificação dos Fatores: $\mathrm{IV}=$ Individualismo Vertical; IH = Individualismo Horizontal; PI = Protoindividualismo; $\mathrm{IE}=$ Individualismo Expressivo $\mathrm{CV}=$ Coletivismo Vertical; $\mathrm{CH}=$ Coletivismo Horizontal. Os coeficientes apresentados em negrito corresponderam aos esperados.

resumidas como segue: o fator individualismo vertical se correlacionou com o atributo orientado ao êxito $(r=0,30, p$ $<0,001)$; o individualismo horizontal o fez com o atributo um ser único $(r=0,30, p<0,001)$; o coletivismo vertical com cumpridor com os demais $(r=0,37, p<0,001)$; o cole- 
tivismo horizontal se correlacionou com o atributo pessoal cooperador $(r=0,24, p<0,001)$; e o protoindividualismo o fez com o atributo batalhador $(r=0,19, p<0,01)$.

Finalmente, procurou-se conhecer em que medida os fatores do individualismo e coletivismo estariam correlacionados com a identificação com os seis endogrupos previamente citados. Como um passo prévio, decidiu-se realizar uma análise de Componentes Principais com as respostas dos participantes aos itens de identificação endogrupal, sendo comprovada a adequação de utilizar tal técnica estatística $(K M O=0,75)$. Os resultados indicaram a presença de dois componentes com eigenvalues superiores a 1,00 , explicando conjuntamente $63,2 \%$ da variância total. A menor carga fatorial foi 0,64 , correspondendo ao item 'familiares em geral'. Os dois componentes, depois da rotação varimax, foram denominados de endogrupos secundários (amigos, companheiros e vizinhos) e endogrupos primários (família, familiares em geral e namorado / esposo); seus Alfas de Cronbach foram 0,71 e 0,59 , respectivamente. A correlação destes itens e fatores com as dimensões do individualismo e coletivismo é apresentada na Tabela 3.

De acordo com esta tabela, a identificação com os diversos endogrupos (pontuação total) se correlacionou mais fortemente com as orientações coletivistas vertical $(r=0,26, p$ $<0,001)$ e horizontal $(r=0,24, p<0,001)$. No caso do individualismo, os fatores que estiveram mais diretamente relacionados com esta pontuação foram o individualismo vertical $(r=0,20, p<0,01)$, o protoindividualismo e o individualismo expressivo $(r=0,19 \mathrm{e} r=0,18$, respectivamente, com $p<0,01$, para ambos); sua dimensão horizontal não se correlacionou significativamente com a identificação endogrupal $(r=0,05, p>0,05)$. Em termos dos dois fatores de endogrupos antes descritos, observou-se o seguinte: o individualismo vertical se correlacionou tanto com aqueles identificados como primários $(r=0,15, p<0,05)$ como secundários $(r=0,17, p<0,01)$; o individualismo horizontal não se correlacionou significativamente com nenhum dos

Tabela 3. Identificação de Individualismo e Coletivismo com Endogrupos

\begin{tabular}{|c|c|c|c|c|c|c|}
\hline \multicolumn{7}{|c|}{ Fatores } \\
\hline & IV & IH & PI & IE & CV & $\mathrm{CH}$ \\
\hline $\begin{array}{l}\text { Identificação com } \\
\text { família }\end{array}$ & 0,06 & $-0,02$ & 0,09 & 0,06 & $0,23 * *$ & $0,25 * *$ \\
\hline $\begin{array}{l}\text { Identificação com } \\
\text { familiares }\end{array}$ & $0,14 *$ & 0,02 & $0,16 * *$ & $0,13^{*}$ & $0,26^{* *}$ & $0,18 * *$ \\
\hline $\begin{array}{l}\text { Identificação com } \\
\text { vizinhos }\end{array}$ & $0,18 * *$ & 0,04 & $0,16 * *$ & $0,14^{*}$ & 0,31 ** & $0,21 * *$ \\
\hline $\begin{array}{l}\text { Identificação com } \\
\text { namorado(a) }\end{array}$ & 0,11 & $-0,06$ & 0,02 & 0,01 & 0,11 & $0,12 *$ \\
\hline Identificação com amigos & 0,09 & $0,14^{*}$ & $0,16 * *$ & $0,14^{*}$ & 0,07 & $0,14^{*}$ \\
\hline $\begin{array}{l}\text { Identificação com } \\
\text { companheiros }\end{array}$ & 0,10 & $0,12 *$ & 0,07 & $0,13^{*}$ & 0,01 & 0,03 \\
\hline Identificação Endogrupal & $0,20 * *$ & 0,05 & $0,19 * *$ & $0,18 * *$ & $0,26^{* * *}$ & $0,24 * * *$ \\
\hline Endogrupos Primários & $0,15^{*}$ & $-0,01$ & 0,11 & 0,09 & $0,26^{* * *}$ & $0,24 * * *$ \\
\hline Endogrupos Secundários & $0,17 * *$ & 0,09 & $0,17 * *$ & $0,19 * *$ & $0,18^{* *}$ & $0,16^{*}$ \\
\hline
\end{tabular}

Notas: * $p<0,05 ; * * p<0,01 ; * * * p<0,001$. Identificação dos fatores: $\mathrm{IV}=$ Individualismo Vertical; $\mathrm{IH}=$ Individualismo Horizontal; PI = Protoindividualismo $; \mathrm{IE}=$ Individualismo Expressivo $; \mathrm{CV}=$ Coletivismo Vertical $; \mathrm{CH}=$ Coletivismo Horizontal. dois $(p>0,05)$; o protoindividualismo o fez unicamente com os endogrupos secundários $(r=0,17, p<0,05)$; este mesmo padrão de correlação foi observado para o fator individualismo expressivo $(r=0,19, p<0,01)$; o coletivismo vertical se correlacionou com os endogrupos primários $(r=0,26, p$ $<0,001)$ e secundários $(r=0,18, p<0,01)$; e o coletivismo horizontal também o fez assim em relação aos dois endogrupos, primários $(r=0,24, p<0,01)$ e secundários $(r=0,16, p$ $<0,05)$.

\section{Discussão}

O propósito principal deste estudo foi apresentar uma medida alternativa dos construtos individualismo e coletivismo, incorporando duas novas dimensões: o protoindividualismo e o individualismo expressivo. Tal medida, provavelmente, é mais adequada para conhecer as facetas que caracterizam dimensões do individualismo que regem a vida dos latinos ou propriamente dos brasileiros. Estes são geralmente motivados por princípios que põem em relevo a harmonia interpessoal, o sentido de cumprimento com os demais e a simpatia (Triandis, Marín, Lisansky, \& Betancourt, 1984), porém não se restringem a uma orientação exclusivamente coletivista. Entre suas prioridades estão também a autodireção e o êxito (Gouveia, 1998), valores que são tipicamente individualistas (Schwartz, 1990). Tal padrão de orientação que mistura valores e atitudes em princípio opostos, que para algumas culturas pareceria anômalo, também tem sido ressaltado por outros pesquisadores (Tamayo, 1997). O protoindividualismo dá conta não dos ideários, mas da real necessidade de sobreviver em um contexto de adversidade e escassez; o individualismo expressivo permite contemplar a importância que se dá aos amigos e companheiros e o desejo de ser reconhecido, ímpar.

Embora se reconheça aqui que o parâmetro de consistência interna (Alfa de Cronbach) não tenha sido o desejável para o protoindividualismo e o individualismo horizontal, deve-se encarar como preliminar a versão ora discutida do instrumento em pauta. Em todo caso, também não se pode perder de vista que cada fator desta medida é composto por apenas cinco itens, que não se repetem ou são redundantes. A consistência interna destes construtos parece ser um problema inerente, sobretudo por reunir em cada dimensão múltiplas idéias e definições, e atributos variados, como atitudes, opiniões, crenças etc. (ver Gouveia \& Clemente, 1998; Triandis, 1995). Além disso, no geral, verificaram-se correlações significativas com os seus respectivos atributos-chave. Por último, os índices de bondade de ajuste, produzidos a partir da análise fatorial confirmatória, apoiam a validade de construto desta medida.

Frente às especulações e os conhecimentos enviesados sobre as dimensões do individualismo e coletivismo (ver Kagitçibasi, 1997), apresenta-se uma tentativa de propor uma medida teoricamente fundamentada. Mais importante, não se encontrou nenhuma outra que tenha sido desenvolvida no contexto latino-americano, onde as informações a respeito destes construtos são escassas, levando a especulações que 
sugerem incluir seus países em um mesmo bloco de culturas coletivistas (Hofstede, 1984; Markus \& Kitayama, 1991). A respeito deste viés ou propriamente estereótipo, o Brasil tem sido caricaturado como coletivista vertical, como o indica Triandis com um exemplo que ele pensa ser típico deste país:

No Brasil, um garçom traz um cardápio para quatro pessoas e o entrega ao membro mais velho do grupo, quem se encarrega de pedir a mesma comida para todos os demais (Triandis, 1995, p. 1).

É possível que esta situação não aconteça com a freqüência que este autor imagina; é certo que economicamente existe desigualdade no Brasil, e que isto compreende um indicador de verticalidade (Triandis \& Gelfand, 1998). Não obstante, talvez este autor esteja exagerando ao oferecer uma visão de extrema obediência à autoridade e conformismo, o que não é o mesmo que coletivismo, ao menos no âmbito conceitual. Definitivamente, as especulações sobre o tipo de orientação dos brasileiros deve ser contrastada com dados de pesquisas empíricas, e neste sentido a Escala Multi-Fatorial de Individualismo e Coletivismo poderia dirimir eventuais equívocos.

Este não é, como se retratou anteriormente, o único instrumento disponível na literatura. Porém, tem a vantagem de apresentar uma estrutura multidimensional, como também o fizeram Singelis e cols. (1995), mas com o diferencial de ser pensado para o contexto cultural brasileiro. A maioria dos demais instrumentos (escalas, questionários), de origem principalmente norte-americana ou asiática, tem enfocado aspectos específicos destas culturas (por exemplo, o ideal de triunfo, a submissão etc.), e negligenciam atributos que nas culturas latinas estão presentes e são fundamentais para explicar o comportamento dos seus membros. Espera-se com o presente instrumento que se abram novas perspectivas de estudo sobre o individualismo e coletivismo. Seria interessante considerar, por exemplo, a relevância do protoindividualismo para explicar condutas típicas do meio rural ou de ambientes de escassez econômica.

Ficou também comprovado que a identificação com os diversos endogrupos é fundamentalmente uma questão de assumir uma orientação coletivista (Gouveia, 1998; Hui, 1988). Adotar atitudes individualistas horizontais significa se manter afastado ou não se identificar com os diversos endogrupos, isto é, assumir-se como um ser único, diferente dos demais (Triandis, 1995). Não obstante, ao menos neste país, os demais fatores do individualismo não implicam uma renúncia ao contato social ou à identificação com endogrupos secundários (amigos, companheiros e vizinhos). Isso reforça, uma vez mais, a particularidade da cultura considerada, em que podem coexistir uma ênfase nas dimensões independente e interdependente da auto-imagem (Singelis, 1994). A pessoa pode ser individualista, mas sem renunciar às suas relações sociais, principalmente àquelas que são estabelecidas no âmbito mais informal, entre os companheiros de estudo ou trabalho.

Finalmente, espera-se que os objetivos deste estudo tenham sido cumpridos, lembrando: propor uma nova medida do individualismo e coletivismo, comprovando sua estrutura com seis fatores, a correlação destes com atributos pessoais que teoricamente os descreveriam e com a identificação endogrupal. Esta medida poderá ser útil em pesquisas futuras, por exemplo, quando se tratar de explicar as relações interpessoais e a identificação com membros de grupos de pertença, atributos que são básicos para entender o comportamento social (Triandis, 1994). Esperar-se-ia no futuro a realização de novos estudos que procurassem aprimorar os fatores medidos, incorporando novos itens e comprovando outros parâmetros psicométricos desta medida, a exemplo da sua estabilidade temporal (teste-reteste) e validade preditiva.

\section{Referências}

Braithwaite, V.A. \& Scott, W. A. (1991). Values. Em J.P. Robinson, P.R. Shaver \& L.S. Wrightsman (Eds.), Measures of personality and social psychological attitudes, vol. 1 (pp. 661 - 753). San Diego, CA: academic Press.

Coelho Júnior, L.L. (2001). Uso potencial de drogas em estudantes do ensino médio: Sua correlação com as prioridades axiológicas. Dissertação de Mestrado, Departamento de Psicologia, Universidade Federal da Paraíba, João Pessoa, PB.

Durkheim, É. (1982). La división del trabajo social. Madri: Akal Editor.(originalmente publucado em 1893).

Feldman, S.S. \& Rosenthal, D.A. (1991). Age expectations of behavioural autonomy in Hong Kong, Australian and American youth: The influence of family variables and adolescents' values. International Journal of Psychology, 26, 1-23.

Gouveia, V.V. (1998). La naturaleza de los valores descriptores del individualismo y del colectivismo: Una comparación intra e intercultural. Tese de Doutorado. Faculdade de Psicologia, Universidade Complutense de Madri, Espanha.

Gouveia, V.V. \& Clemente, M. (1998). La medida del individualismo y del colectivismo: La investigación en el campo de la Psicología Cultural. La Coruña: Editorial Universidad de La Coruña.

Gouveia, V.V. \& Clemente, M. (2000). O individualismo-coletivismo no Brasil e na Espanha: Correlatos sócio-demográficos. Estudos de Psicologia, 5, 317-346.

Gouveia, V.V., Clemente, M. \& Espinosa, P. (2001). The horizontal and vertical attributes of individualism and collectivism: Replicating the Singelis et al.'s study in Spain. The Journal of Social Psychology (no prelo).

Gouveia, V.V. \& Ros, M. (2000). Hofstede's and Schwartz's models for classifying individualism at the cultural level: Their relation to macro-social and macro-economic variables. Psicothe$m a, 1,25-33$.

Gusfield, J.R. (1967). Tradition and modernity: Misplaced polarities in the study of social change. American Journal of Sociology, 73, 351-362.

Hofstede, G. (1984). Culture's consequences: International differences in work-related values. Beverly Hills, CA: Sage Publications.

Hofstede, G. (1994). Foreword. Em U. Kim, H.C. Triandis, Ç. Kagitçibasi, S.-C. Choi \& G. Yoon (Eds.), Individualism and 
collectivism: Theory, method, and applications (pp. ix-xii). Thousand Oaks, CA: Sage Publications.

Hofstede, G. \& Bond, M.H. (1984). Hofstede's culture dimensions: An independent validation using Rokeach's Value Survey. Journal of Cross-Cultural Psychology, 15, 417-433.

Hui, C.H. (1988). Measurement of individualism-collectivism. Journal of Research in Personality, 22, 17-36.

Hui, C.H. \& Yee, C. (1994). The shorted Individualism-Collectivism Scale: Its relationship to demographic and work-related variables. Journal of Research in Personality, 28, 409-424.

Imamoglu, E.O. (1998). Individualism and collectivism in a model and scale of balanced differentiation and integration. The Journal of Psychology, 132, 95-105.

Jöreskog, K.G. \& Sörbom, D. (1989). LISREL 7 user's reference guide. Mooresville, IN: Scientific Software.

Kagitcibasi, C. (1994). A critical appraisal of individualism and collectivism: Toward a new formulation. Em U. Kim, H.C. Triandis, Ç. Kagitçibasi, S.-C. Choi \& G. Yoon (Eds.), Individualism and collectivism: Theory, method, and applications (pp. 52-65). Thousand Oaks, CA: Sage Publications.

Kagitcibasi, Ç. (1997). Individualism and collectivism. Em J.W. Berry, M.H. Segall \& Ç. Kagitçibasi (Eds.), Handbook of crosscultural psychology. Social behavior and applications, vol. 3 (pp. 1-49). Boston: Allyn and Bacon.

Lonner, W.J. \& Berry, J.W. (1994). Series editors' introduction. Em U. Kim, H.C. Triandis, Ç. Kagitçibasi, S.-C. Choi \& G. Yoon (Eds.), Individualism and collectivism: Theory, method, and applications (pp. xv-xvi). Thousand Oaks, CA: Sage Publications.

Maia, L.M.M. (2000). Prioridades valorativas e desenvolvimento moral: Considerações acerca de uma teoria dos valores humanos. Dissertação de Mestrado, Departamento de Psicologia, Universidade Federal da Paraíba, João Pessoa, PB.

Markus, H.R. \& Kitayama, S. (1991). Culture and the self: Implications for cognition, emotion, and motivation. Psychological Review, 98, 224-253.

Moorman, R.H. \& Blakely, G.L. (1995). Individualism-collectivism as an individual difference predictor of organizational citizenship behavior. Journal of Organizational Behavior, 16, $127-$ 142.

Morales, J.F., López, M. \& Veja, L. (1992). Individualismo, colectivismo e identidad social. Revista de Psicología Social, Monográfico, 49-72.

Parsons, T. (1976). El sistema social. Madri: Revista de Occidente (originalmente publicado em 1959).

Rhee, E., Uleman, J.S. \& Lee, H.K. (1996). Variations in collectivism and individualism by ingroup and culture: Confirmatory factor analysis. Journal of Personality and Social Psychology, $71,1037-1054$.

Schwartz, S.H. (1990). Individualism-collectivism: Critique and proposed refinements. Journal of Cross-Cultural Psychology, 21, 139-157.

Singelis, T.M. (1994). The measurement of independent and interdependent self-construals. Personality and Social Psychological Bulletin, 20, 580-591.

Singelis, T.M., Triandis, H.C., Bhawuk, D.S. \& Gelfand, M.J. (1995). Horizontal and vertical dimensions of individualism and collectivism: A theoretical and measurement refinement. Cross-Cultural Research, 29, 240-275.

Sinha, D. \& Tripathi, R.C. (1994). Individualism in a collectivist culture: A case of coexistence of opposites. Em U. Kim, H.C. Triandis, Ç. Kagitçibasi, S.-C. Choi \& G. Yoon (Eds.), Individualism and collectivism: Theory, method, and applications (pp. 123-136). Thousand Oaks, CA: Sage Publications.

Tamayo, A. (1997). Os valores do brasileiro: Uma década de pesquisa. Cadernos de Psicologia, 1, 115-134.

Tönnies, F. (1979). Comunidady asociación. Barcelona: Ediciones Península.(originalmente publicado em 1887)

Touraine, A. (1993). Crítica de la modernidade. Madri: Ediciones Temas de Hoy.

Triandis, H.C. (1988). Cross-cultural contributions to theory in social psychology. Em M.H. Bond (Ed.), The cross-cultural challenge to social psychology (pp. 122-140). Newbury Park, CA: Sage Publications.

Triandis, H.C. (1993). Collectivism and individualism as cultural syndromes. Cross-Cultural Research, 27, 155-180.

Triandis, H.C. (1994). Culture and social behavior. New York: McGraw-Hill.

Triandis, H.C. (1995). Individualism and collectivism. Boulder, CO: Westview Press.

Triandis, H.C. \& Gelfand, M.J. (1998). Converging measurement of horizontal and vertical individualism and collectivism. Journal of Personality and Social Psychology, 74, 118-128.

Triandis, H.C., Marín, G., Lisansky, J. \& Betancourt, H. (1984). Simpatía as a cultural script of hipanics. Journal of Personality and Social Psychology, 47, 1363-1375.

Triandis, H.C., Bontempo, R., Betancourt, H., Bond, M., Leung, K., Brenes, A., Georgas, J., Hui, C. H., Marin, G., Setiadi, B., Sinha, J.B.P., Verma, J., Spangenberg, J., Touzard, H. \& Montmollin, G. (1986). The measurement of the etics aspects of individualism and collectivism across cultures. Australian Journal of Psychology, 38, 257-267.

Van de Vijver, F. \& Leung, K. (1997). Methods and data analysis for cross-cultural research. Thousand Oaks, CA: Sage Publications.

Yamaguchi, S. (1994). Collectivism among the Japanese: A perspective from the self. Em U. Kim, H.C. Triandis, Ç. Kagitçibasi, S.-C. Choi \& Yoon (Eds.), Individualism and collectivism: Theory, method, and applications (pp. 175-188). Thousand Oaks, CA: Sage Publications.

Wagner, J.A. III \& Moch, M.K. (1986). Individualism-collectivism: Concept and measure. Group \& Organization Studies, 11, 280-304. 